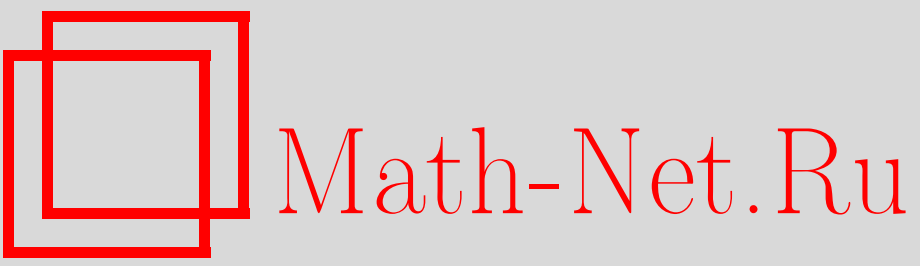

А. Н. Куликов, Инерциальные инвариантные многообразия нелинейной полугруппы операторов в гильбертовом пространстве, Итоги науки и техн. Сер. Соврем. мат. и ее прил. Темат. обз., 2020, том 186, 57-66

DOI: https://doi.org/10.36535/0233-6723-2020-186-57-66

Использование Общероссийского математического портала Math-Net.Ru подразумевает, что вы прочитали и согласны с пользовательским соглашением

http://www.mathnet.ru/rus/agreement

Параметры загрузки:

IP : 54.197 .130 .99

26 апреля 2023 г., 04:04:56 


\title{
ИНЕРЦИАЛЬНЫЕ ИНВАРИАНТНЫЕ МНОГООБРАЗИЯ НЕЛИНЕЙНОЙ ПОЛУГРУППЫ ОПЕРАТОРОВ В ГИЛЬБЕРТОВОМ ПРОСТРАНСТВЕ
}

\author{
(C) 2020 г. \\ А. Н. КУЛИКОВ
}

\begin{abstract}
АннотАция. Изучены вопросы о существовании и свойствах инерциальных многообразий нелинейной полугруппы операторов в гильбертовом пространстве. Данный круг вопросов изучен в достаточно общей постановке, позволяющей обобщить результаты известных работ К. Фояша, Дж. Селла и Р. Темама. Для изучения вопроса о существовании и свойствах использована схема доказательств соответствующих утверждений, предложенных в свое время С. Стернбергом и Ф. Хартманом при изучении аналогичных вопросов для обыкновенных автономных дифференциальных уравнений.
\end{abstract}

Ключевые слова: инерциальное инвариантное многообразие, гильбертово пространство, оператор.

\section{INERTIAL INVARIANT MANIFOLDS \\ OF A NONLINEAR SEMIGROUP OF OPERATORS \\ IN A HILBERT SPACE}

\author{
(c) 2020 A. N. KULIKOV
}

\begin{abstract}
In this paper, we examine the existence and analyze properties of inertial manifolds of a nonlinear semigroup of operators in a Hilbert space. This questions were studied in a general setting that allows generalizing results of the well-known works of K. Foias, J. Sell, and R. Temam. Our reasoning is based on the scheme of proofs of similar assertions proposed earlier by S. Sternberg and F. Hartman for ordinary autonomous differential equations.
\end{abstract}

Keywords and phrases: inertial invariant manifold, Hilbert space, operator.

AMS Subject Classification: 37L25, 37L05

1. Введение. В вещественном гильбертовом пространстве $H$ рассмотрим полугруппу нелинейных операторов

$$
Z(t, u)=T(t) u+R(t, u)
$$

Будем предполагать, что $T(t)$ - полугруппа линейных ограниченных операторов класса $\left(C_{0}\right)$ [4]. Производящий оператор полугруппы $T(t)$, область определения которого $H_{A}$ плотна в $H$, обозначим $A$.

Пусть $\lambda_{j}=\alpha_{j}+i \beta_{j}(j=1,2,3, \ldots)-\mathrm{C} 3$ линейного оператора $A$. Через $e_{j}=h_{j}+i g_{j}$ обозначим соответствующие им собственные и присоединенные элементы. При рассмотрении вопроса о собственных значениях и собственных элементах линейного оператора $A$ в ситуации общего положения следует рассматривать этот оператор в комплексном расширении пространства $H$. Через 
$\varphi_{j}=\psi_{j}+i \Theta_{j}$ обозначим собственные и присоединенные элементы сопряженного оператора $A$, отвечающие тому же собственному значению $\lambda_{j}$. Будем считать, что они образуют биортогональные системы и обе они являются базисом Рисса (Рисса-Бари) (см., например, $[1,13])$.

Собственные числа линейного оператора $A$ считаем пронумерованными таким образом, что справедлива цепочка неравенств

$$
\alpha_{1} \geqslant \alpha_{2} \geqslant \alpha_{3} \geqslant \ldots \geqslant \alpha_{j} \geqslant \ldots
$$

Предположим также, что можно указать такое натуральное $N$, что

1. $\alpha_{N}-\alpha_{N+1}=\alpha>0$;

2. $\alpha_{N+1}<0$.

В силу предположений можно считать, что любой элемент $f \in H$ допускает представление в виде

$$
f=\sum_{j=1}^{\infty} f_{j} e_{j}, \quad f_{j}=\left(f, \varphi_{j}\right),
$$

где $(*, *)$ - скалярное произведение в $\overline{H_{c}}$, где через $H_{c}$ обозначено комплексное расширение $H$. При этом $f_{k}=\bar{f}_{j}$, если $e_{k}$ и $e_{j}$ соответствуют комплексно сопряженным собственным значениям $\lambda_{k}, \lambda_{j}\left(\lambda_{k}=\bar{\lambda}_{j}\right)$. Также считаем, что все С3 $\lambda_{j}$ имеют конечную кратность $k_{j}$ и, более того, $k_{j} \leqslant$ $k_{\max }$, где $k_{\max }$ - некоторое натуральное число. Далее будем использовать обозначение $\|u\|_{A}=$ $\|u\|+\|A u\|$, если $u \in H_{A}$, а $\|\cdot\|-$ норма в $H$.

Перейдем к описанию нелинейного оператора $R(t, u)$. Пусть выполнены следующие свойства:

1. $R(t, 0)=0$ при всех $t$;

2. нелинейный оператор $R(t, u)$ непрерывен по совокупности переменных в норме $H_{A}$, как оператор из $\mathbb{R}_{+}^{1} \times H_{A}$ в $H_{A}\left(\mathbb{R}_{+}^{1}=\{t \geqslant 0\}\right)$;

3. $\|R(t, u)\|_{A} \leqslant a_{0}(t)$;

4. $\|R(t, u)-R(t, v)\|_{A} \leqslant a_{1}(t)\|u-v\|_{A}$.

Здесь $a_{0}(t), a_{1}(t)$ - непрерывно дифференцируемые функции. При этом $a_{0}(t), a_{1}(t) \geqslant 0$, а также $a_{0}(t), a_{1}(t) \rightarrow 0$, если $t \rightarrow 0$. Пусть, кроме того, $\dot{a}_{1}(0)=K$, где положительная постоянная $K$ достаточно мала. Степень малости укажем ниже, то есть $K$ считаем «регулируемым параметром».

Пусть $H_{1}$ - подпространство $H$, натянутое на элементы

$$
h_{1}, h_{2}, \ldots, h_{N}, g_{1}, g_{2}, \ldots, g_{N}, H_{1 A}=H_{1} \cap H_{A} .
$$

Ясно, что $H_{1 A}=H_{1}\left(\operatorname{dim} H_{1}=n\right)$ и $H_{1}$ изоморфно $\mathbb{R}^{n}$, где $n \leqslant 2 N$. Обозначим через $P_{1}$ проектор на $H_{1}$ и положим $P_{2}=I-P_{1}$, где $I$ - единичный оператор. Положим $H_{2}=P_{2} H, H_{2 A}=$ $P_{2} H_{A}$. Тогда, используя введенные проекторы и подпространства, нелинейную полугруппу (1) можно переписать в следующем виде

$$
\begin{gathered}
v_{t}=T_{1}(t) v+R_{1}(t, v, w), \\
w_{t}=T_{2}(t) v+R_{2}(t, v, w), \\
v_{t}=P_{1} u(t), \quad w_{t}=P_{2} u(t), \quad v=P_{1} u, \quad w=P_{2} w, \\
R_{1}=P_{1} R, \quad R_{2}=P_{2} R, \quad T_{1}(t)=P_{1} T(t), \quad T_{2}(t)=P_{2} T(t) .
\end{gathered}
$$

Семейство операторов $\left\{T_{1}(t)\right\}$ состоит из линейных операторов, действующих в конечномерном пространстве $H_{1}\left(\mathbb{R}^{n}\right)$. Они образуют уже группу линейных ограниченных операторов. При этом $T_{1}(-t)=T_{1}^{-1}(t)$. Добавим, что группа $T_{1}(t)$ порождена решениями системы линейных обыкновенных дифференциальных уравнений

$$
\dot{v}=A_{1} v, \quad A_{1}=P_{1} A, \quad v \in H_{1}=\mathbb{R}^{n}, \text { т. е. } v=P_{1} u,
$$

то есть фактически совпадает с матричной экспонентой $\exp \left(A_{1} t\right)$, если оператор $A_{1}$ записать в виде матрицы. Добавим, что в подпространствах $H_{1}, H_{2 A}$ можно ввести нормы таким образом, что справедливы следующие оценки

$$
\exp \left(\gamma_{2} t\right) \leqslant\left\|T_{1}(t)\right\| \leqslant \exp \left(\gamma_{1} t\right), \quad\left\|T_{2}(t)\right\| \leqslant \exp \left(\gamma_{3} t\right),
$$


где $\gamma_{1}=\alpha_{1}+\delta, \gamma_{2}=\alpha_{N}-\delta, \gamma_{3}=\alpha_{N+1}+\delta$, а $\delta$, в свою очередь, достаточно малая положительная постоянная. Через $\left\|T_{j}(t)\right\|, j=1,2$, обозначены нормы в пространстве ограниченных линейных операторов, индуцированные нормами в подпространствах $H_{1}, H_{2}$. В силу произвольности положительной постоянной $\delta$ без нарушения общности можно считать, что $\delta \leqslant K$.

Возможность введения норм, в которых справедливы оценки (5), хорошо известна для конечномерных подпространств [15]. В нашем случае такую возможность гарантирует конечномерность корневых подпространств, соответствующих каждому из С3 $\lambda_{k}$, а также то обстоятельство, что размерность каждого из них ограничена числом $k_{\max }$. Добавим, что в ситуации, когда все $\lambda_{k}$ были бы простыми С3 соответствующего оператора, то корневые подпространства были бы одномерными, и можно было бы выбрать $\delta=0$.

Предположение о конечной кратности собственных значений не является излишне обременительным, так как в подавляющем числе примеров выполняется. С другой стороны, если некоторое собственное число имеет бесконечную кратность или есть последовательность чисел $\left\{\lambda_{m}\right\}$, кратность которых $k_{m} \rightarrow \infty$ при $m \rightarrow \infty$, то в обоих случаях, как хорошо известно, оценка нормы полугруппы [16]

$$
\|T(t)\| \leqslant M \exp (\omega t), \quad M>0, \quad \omega \in \mathbb{R},
$$

носит академический характер и не всегда связана с расположением собственных чисел производящего оператора данной полугруппы [20,22].

Напомним некоторые определения, которые в дальнейшем будут использованы (см., например, $[10,18,21])$.

Определение 1. Множество (многообразие) $M$ называется инвариантным для нелинейной полугруппы (1), если включение $u \in M$ влечет за собой включение $Z(t, u) \in M$ при всех $t \geqslant 0$.

Определение 2. Множество (многообразие) $M_{l}$ называется локально инвариантным, если оно инвариантно для семейства отображений (1) при всех $t \geqslant 0$ пока $Z(t, u) \in S_{A}(r)$ при некотором $r>0$. Здесь $S_{A}(r)$ - шар радиуса $r$ в пространстве $H_{A}$, т. е. $u \in S_{A}(r)$, если $\|u\|_{A} \leqslant r$.

Определение 3. Следуя работе [18], инвариантное многообразие (множество) $M_{\text {in }}$ будем называть инерциальным многообразием, если

1. $M$ - конечномерно;

2. $M$ - экспоненциальный аттрактор для нелинейной полугруппы (1), т. е. справедливо предельное равенство $\lim _{t \rightarrow \infty} d\left(Z(t, u), M_{i n}\right)=0$. При этом для функции $d(t)=d\left(Z(t, u), M_{i n}\right)$ справедлива экспоненциальная оценка

$$
d(t) \leqslant C \exp (-\nu t), \quad C, \nu>0 .
$$

Определение 4. Инвариантное многообразие $M \subset C_{Z}$, если оно задается уравнением

$$
w_{t}=V\left(v_{t}\right)
$$

где нелинейный оператор $V(v)$ действует из $H_{1}$ в $H_{2 A}=H_{A} \cap H_{2}$. При этом он обладает следующими свойствами:

1. $V(0)=0$;

2. $\left\|V\left(v_{1}\right)-V\left(v_{2}\right)\right\|_{A} \leqslant\left\|v_{1}-v_{2}\right\|_{A}$;

3. нелинейный оператор $V(v)$ определен при всех $v \in H_{A}$ и для него определена норма

$$
\|V(v)\|=\sup _{v \in H_{A}}\|V(v)\|_{A} .
$$

Далее через $d\left(V_{1}, V_{2}\right)$ будем обозначать расстояние в пространстве $C_{Z}$

$$
d\left(V_{1}, V_{2}\right)=\left\|V_{1}(v)-V_{2}(v)\right\| .
$$

Теорема 1. Пусть выполнены неравенства

$$
-\alpha+6 K<0, \quad \alpha_{N+1}+2 K<0,
$$

то есть $K \leqslant \min \left(\alpha / 6,-\alpha_{N+1} / 2\right)$. Тогда нелинейнал полугруппа (1) имеет единственное инерииальное многообразие $M_{\text {in }} \subset C_{Z}$. 
Отметим, что неравенство $K<\min \left(\alpha / 6, \alpha_{N+1} / 2\right)$ выполнено в силу предположения, что $K$ достаточно малая постоянная.

Теорема 2. При $t \geqslant 0$ справедливо неравенство

$$
d\left(Z(t, u), M_{i n}\right) \leqslant K_{0} \exp \left(-\gamma_{0} t\right) d\left(u, M_{i n}\right),
$$

где $K_{0}, \gamma_{0}$ - положительные постоянные, а через $d\left(u, M_{i n}\right)$ обозначено расстояние от элемента и до $M_{\text {iп }}$ в смысле метрики пространства $H_{A}$.

Напомним, что $d\left(y, M_{i n}\right)=\inf _{y_{i n} \in M_{i n}}\left\|y-y_{i n}\right\|_{A}$.

2. Доказательство теоремы о существовании инерциального многообразия. Пусть $V(v) \in C_{Z}$. Рассмотрим вспомогательное отображение $\mathbb{R}^{n}$ в $\mathbb{R}^{n}:$

$$
v_{t}=B(t, v, V)=T_{1}(t) v+R_{1}(t, v, V(v)),
$$

которое, конечно, зависит от выбора $V=V(v) \in C_{Z}$.

Лемма 1. Справедливы неравенства

$$
\begin{gathered}
\left\|v_{t}^{(1)}-v_{t}^{(2)}\right\|_{A} \leqslant a_{2}(t)\left\|v^{(1)}-v^{(2)}\right\|_{A}, \\
\left\|v^{(1)}-v^{(2)}\right\|_{A} \leqslant a_{3}(t)\left\|v_{t}^{(1)}-v_{t}^{(2)}\right\|_{A},
\end{gathered}
$$

если $t \in\left(0, t_{1}\right)$. Здесь

$$
\begin{gathered}
a_{2}(t)=\exp \left(\gamma_{1} t\right)+2 a_{1}(t), \quad a_{3}(t)=\frac{\exp \left(-\gamma_{2} t\right)}{\left(1-a_{1}(t) \exp \left(-\gamma_{2} t\right)\right)} \\
\dot{a}_{2}(0)=\gamma_{1}+2 K, \quad \dot{a}_{3}(0)=-\gamma_{2}+2 K
\end{gathered}
$$

Действительно,

$$
\left(v_{t}^{(1)}-v_{t}^{(2)}\right)=T_{1}(t)\left(v^{(1)}-v^{(2)}\right)+\left(R_{1}\left(t, v^{(1)}, V\left(v^{(1)}\right)\right)-R_{1}\left(t, v^{(2)}, V\left(v^{2}\right)\right) .\right.
$$

Откуда

$$
\begin{aligned}
\left\|v_{t}^{(1)}-v_{t}^{(2)}\right\|_{A} & \leqslant\left\|T_{1}(t)\right\|\left\|v^{(1)}-v^{(2)}\right\|_{A}+\left\|R_{1}\left(t, v^{(1)}, V\left(v^{1}\right)\right)-R_{1}\left(t, v^{(2)}, V\left(v^{2}\right)\right)\right\|_{A} \leqslant \\
& \leqslant \exp \left(\gamma_{1} t\right)\left\|v^{(1)}-v^{(2)}\right\|_{A}+a_{1}(t)\left\|v^{(1)}-v^{(2)}\right\|_{A}+a_{1}(t) \| V\left(v^{(1)}\right)-V\left(v^{(2)} \|_{A} \leqslant\right. \\
& \leqslant \exp \left(\gamma_{1} t\right)\left\|v^{(1)}-v^{(2)}\right\|_{A}+a_{1}(t)\left\|v^{(1)}-v^{(2)}\right\|_{A}+a_{1}(t)\left\|v^{(1)}-v^{(2)}\right\|_{A} \leqslant \\
& \leqslant\left[\exp \left(\gamma_{1} t\right)+2 a_{1}(t)\right]\left\|v^{(1)}-v^{(2)}\right\|_{A} .
\end{aligned}
$$

Неравенство (7) доказано. При доказательстве неравенства (7) было использовано включение $V(v) \in C_{Z}$. В частности, неравенство

$$
\left\|V\left(v^{(1)}\right)-V\left(v^{(2)}\right)\right\|_{A} \leqslant\left\|v^{(1)}-v^{(2)}\right\|_{A} .
$$

Перейдем к доказательству неравенства (8). Очевидно, что

$$
\begin{aligned}
\left\|v^{(1)}-v^{(2)}\right\|_{A} \leqslant\left\|T_{1}(t)^{-1}\right\|\left(\|\left(v_{t}^{(1)}-v_{t}^{(2)}\right)-R_{1}\left(t, v_{t}^{(1)}, V\left(v^{(1)}\right)\right)-\right. & \\
& \left.-R_{2}\left(t, v_{t}^{(2)}, V\left(v^{(2)}\right)\right) \|_{A}\right) \leqslant \exp \left(-\gamma_{2} t\right)\left(\left\|v_{t}^{(1)}-v_{t}^{(2)}\right\|_{A}+2 a_{1}(t)\left\|v^{(1)}-v^{(2)}\right\|_{A}\right) .
\end{aligned}
$$

Откуда получаем, что

$$
\left\|v^{(1)}-v^{(2)}\right\|_{A} \leqslant a_{3}(t)\left\|v_{t}^{(1)}-v_{t}^{(2)}\right\|_{A},
$$

где $a_{3}(t)=\exp \left(-\gamma_{2} t\right) /\left(1-2 a_{1}(t) \exp \left(-\gamma_{2} t\right)\right)$. При выводе неравенства (8) использовано условие $1-2 a_{1}(t) \exp \left(-\gamma_{2} t\right)>0$. Это означает, что $t_{1}$ следует выбрать так, чтобы последнее неравенство было выполнено при $t \in\left[0, t_{1}\right]$. Существование такого $t_{1}$ гарантирует условие $a_{1}(0)=0$. Проверка формул для $\dot{a}_{2}(0), \dot{a}_{3}(0)$ использует равенства $a_{1}(0)=0, \dot{a}_{1}(0)=K$.

Лемма 2. Существует такое $t_{2}>0$, ито при $t \in\left[0, t_{2}\right]$ отображение (6) обратимо при любом выборе $V(v) \in C_{Z}$

$$
v=B^{-1}\left(t, v_{t}, V\right) \text {. }
$$


Рассмотрим равенство (6) как уравнение для определения $v$. Его решение будем искать в виде

$$
v=T_{1}^{-1}(t)\left(v_{t}+y\right) .
$$

В результате последней подстановки получим равенство

$$
\begin{gathered}
y=R_{0}(t, y), \\
R_{0}(t, y)=-R_{1}\left(t, T_{1}^{-1}(t)\left(v_{t}+y\right), V\left(T_{2}^{-1}(t)\left(v_{t}+y\right)\right)\right) .
\end{gathered}
$$

Уравнение (9) имеет единственное решение, так как оператор $R_{0}(t, y)$ по переменной $y$ является оператором сжатия. Действительно,

$$
\begin{aligned}
& \| R_{0}\left(t, y^{(1)}-R_{0}\left(t, y^{(2)}\right)\left\|_{A} \leqslant a_{1}(t)\right\| T_{1}^{-1}(t)\left(y^{(1)}-y^{(2)}\right) \|_{A}+\right. \\
& \quad+a_{1}(t)\left\|V\left(T^{-1}\left(v_{t}+y^{(1)}\right)\right)-V\left(T_{1}^{-1}\left(v_{t}+y^{(2)}\right)\right)\right\|_{A} \leqslant 2 a_{1}(t) \exp \left(-\gamma_{2} t\right)\left\|y^{1}-y^{2}\right\|_{A} .
\end{aligned}
$$

Положим $a_{4}(t)=2 a_{1}(t) \exp \left(-\gamma_{2} t\right)$. Существует такая положительная постоянная $t_{3}$, что при всех $t \in\left[0, t_{3}\right]$ справедливо $a_{4}(t)<1$. При выборе $t_{3}>0$ еще раз использовано условие $a_{1}(0)=0$.

Лемма 3. Пусть $V_{k}(v) \in C_{Z}, k=1,2$. Положим $v^{(k)}=B^{-1}\left(t, v_{t}, V_{k}\right)$. Тогда при $t \in\left[0, t_{1}\right]$ справедливо неравенство

$$
\begin{gathered}
\left\|v^{(1)}-v^{(2)}\right\|_{A} \leqslant a_{5}(t) d\left(V_{1}, V_{2}\right), \\
a_{5}(t)=a_{1}(t) \frac{\exp \left(-\gamma_{2} t\right)}{1-2 a_{1}(t) \exp \left(-\gamma_{2} t\right)}, \quad a_{5}(0)=0, \quad \dot{a}_{5}(0)=K .
\end{gathered}
$$

Доказательство практически дословно повторяет построения при проверке справедливости утверждения из предыдущей леммы.

Перейдем непосредственно к доказательству теоремы 1 . Пусть при некотором $t>0$ отображение (2) имеет инвариантное многообразие $M_{t} \in C_{Z}$ и оно задано уравнением

$$
w=V_{t}(v)\left(V_{t}(v) \in C_{Z}\right) .
$$

Введем новые переменные $x=v, y=w-V_{t}(v)$. Такая замена обратима $v=x, w=x+V_{t}(x)$. В переменных $x, y$ отображение (2) перепишется следующим образом:

$$
x_{t}=T_{1}(t) x+X(t, x, y), \quad y_{t}=T_{2}(t) y+Y(t, x, y),
$$

где

$$
\begin{aligned}
& X(t, x, y)=R_{1}\left(t, x, y+V_{t}(x)\right), \\
& Y(t, x, y)=T_{2}(t) V_{t}(v)+R_{2}\left(t, x, y+V_{t}(x)\right)-V_{t}\left(T_{1}(t) x+X(t, x, y)\right) .
\end{aligned}
$$

В переменных $x, y$ инвариантное многообразие $M_{t}$ задается равенством $y=0$. Это означает, что нелинейный оператор $V_{t}(x)$ следует искать как решение операторного уравнения

$$
V_{t}\left(x_{t}\right)=T_{2}(t) V_{t}(x)+R_{2}\left(t, x, V_{t}(x)\right)
$$

где $x, x_{t}$ связаны соотношением $x_{t}=T_{1}(t) x+R_{1}\left(t, x, V_{t}(x)\right)$. В результате для $V_{t}$ получаем операторное уравнение

$$
V_{t}(x)=G_{t} V_{t}(x)
$$

где нелинейный оператор $G_{t}$ определен равенством

$$
G_{t} V_{t}(x)=T_{2}(t) V_{t}\left(B^{-1}\left(t, x, V_{t}\right)\right)+R_{2}\left(t, B^{-1}\left(t, x, V_{t}\right), V_{t}\left(B^{-1}, t, x, V_{t}\right)\right) .
$$

В последних двух формулах $x_{t}$ заменено на $x B^{-1}\left(t, x_{t}, V_{t}\right) \rightarrow B^{-1}\left(t, x, V_{t}\right)$ для упрощения записи.

Проверим сначала, что нелинейный оператор $G_{t}$ приводит нелинейный оператор $V_{t}(x) \in C_{Z}$ в нелинейный оператор того же класса.

Положим $\bar{V}_{t}=G_{t} V_{t}(x)$, где $V_{t}(x) \in C_{Z}$. Очевидно, что $\bar{V}_{t}(0)=0$. Действительно, справедлива цепочка равенств

$$
V_{t}(0)=0, B^{-1}\left(t, 0, V_{t}\right)=0, R_{1}(t, 0,0)=0 .
$$


Заметим также, что

$$
\left.\left\|\bar{V}_{t}(x)\right\|_{A} \leqslant \exp \left(\gamma_{3} t\right) \| V_{t}\left(B^{-1}\left(t, x, V_{t}\right)\right)\right) \|_{A}+a_{0}(t) \leqslant \bar{M}_{0}(t),
$$

если $\left\|V_{t}(x)\right\|_{A} \leqslant M_{0}(t)$. Наконец,

$$
\left\|\overline{V_{t}}\left(x_{1}\right)-\overline{V_{t}}\left(x_{2}\right)\right\| \leqslant\left\|T_{2}(t)\right\|\left\|V_{t}\left(x^{(1)}\right)-V_{t}\left(x^{(2)}\right)\right\|_{A}+\left\|R_{2}\left(t, x^{(1)}, V_{t}\left(x^{(1)}\right)\right)-R_{2}\left(t, x^{(2)}, V_{t}\left(x^{(2)}\right)\right)\right\|_{A},
$$

где $x^{(k)}=B^{-1}\left(t, x_{k}, V_{t}\right)$. Отметим также, что

$$
\left\|x^{(1)}-x^{(2)}\right\|_{A} \leqslant a_{3}(t)\left\|x_{1}-x_{2}\right\|_{A}
$$

в силу результатов из лемм 2,3 и переобозначений. Поэтому

$$
\left\|\bar{V}_{t}\left(x_{1}\right)-\bar{V}_{t}\left(x_{2}\right)\right\| \leqslant \exp \left(\gamma_{3} t\right) a_{3}(t)\left\|x_{1}-x_{2}\right\|_{A}+2 a_{1}(t) a_{3}(t)\left\|x_{1}-x_{2}\right\|_{A}=q_{1}(t)\left\|x_{1}-x_{2}\right\|_{A} .
$$

Здесь $q_{1}(t)=a_{3}(t)\left(\exp \left(\gamma_{3} t\right)+2 a_{1}(t)\right)$. При этом

$$
q_{1}(0)=1, \quad \dot{q}_{1}(0)=\gamma_{3}+4 K-\gamma_{2} \leqslant \alpha_{N+1}-\alpha_{N}+2 \delta+4 k \leqslant \alpha_{N+1}-\alpha_{N}+6 K<0
$$

в силу предположений (см. введение). Поэтому при $t \in\left[0, t_{5}\right]$ справедливо неравенство $q_{1}(t) \leqslant 1$, а при $t \in\left(0, t_{5}\right]$ уже строгое неравенство $q_{1}(t)<1$. Последние два неравенства гарантируют выполнение неравенства $\left\|\bar{V}_{t}\left(x_{1}\right)-\bar{V}_{t}\left(x_{2}\right)\right\|_{A} \leqslant\left\|x_{1}-x_{2}\right\|_{A}$. Итак, $\bar{V}_{t} \in C_{Z}$, если $V_{t}(x) \in C_{Z}$.

Лемма 4. Существует такое $t_{6}>0$, что при всех $t \in\left(0, t_{6}\right] G_{t}$-оператор сжатия.

Для доказательства леммы 4 достаточно проверить справедливость неравенства

$$
d\left(\bar{V}_{1 t}, \bar{V}_{2 t}\right) \leqslant q_{2}(t) d\left(V_{1 t}, V_{2 t}\right),
$$

где $\bar{V}_{k t}=G_{t} V_{k t}, k=1,2$, а $q_{2}(t)<1$, если $t \in\left(0 ; t_{6}\right]$.

Из равенства (10) получаем, что

$$
\left\|\bar{V}_{1 t}(x)-\bar{V}_{2 t}(x)\right\|_{A} \leqslant \exp \left(\gamma_{3} t\right)\left\|V_{1 t}\left(x_{1}\right)-V_{2 t}\left(x_{2}\right)\right\|_{A}+\left\|R_{2}\left(t, x_{1}, V_{1 t}\left(x_{1}\right)\right)-R_{2}\left(t, x_{2}, V_{2 t}\left(x_{2}\right)\right)\right\|_{A} .
$$

В данном случае $x_{1}=B^{-1}\left(t, x, V_{1 t}\right), x_{2}=B^{-1}\left(t, x, V_{2 t}\right)$. Напомним, что из леммы 3 вытекает справедливость неравенства

$$
\left\|x_{1}-x_{2}\right\|_{A} \leqslant a_{5}(t) d\left(V_{1 t}, V_{2 t}\right) .
$$

Следовательно, из неравенства (12) вытекает, что

$$
\begin{aligned}
& \left\|\bar{V}_{1 t}-\bar{V}_{2 t}\right\|_{A} \leqslant\left\|T_{2}^{-1}(t)\right\|\left(\left\|V_{1 t}\left(x_{1}\right)-V_{1 t}\left(x_{2}\right)\right\|+\left\|V_{1 t}\left(x_{2}\right)-V_{2 t}\left(x_{2}\right)\right\|_{A}\right)+ \\
& +\left\|R_{2}\left(t, x_{1}, V_{1 t}\left(x_{1}\right)\right)-R_{2}\left(t, x_{2}, V_{2 t}\left(x_{1}\right)\right)\right\|_{A}+\left\|R_{2}\left(t, x_{2}, V_{1 t}\left(x_{1}\right)\right)-R_{2}\left(t, x_{2}, V_{2 t}\left(x_{2}\right)\right)\right\|_{A} \leqslant \\
& \quad \leqslant \exp \left(\gamma_{3} t\right)\left[a_{5}(t)+1\right] d\left(V_{1 t}, V_{2 t}\right)+a_{1}(t) a_{5}(t) d\left(V_{1 t}, V_{2 t}\right)+a_{1}(t)\left(1+a_{5}(t)\right) d\left(V_{1 t}, V_{2 t}\right) .
\end{aligned}
$$

Здесь были использованы результаты предыдущих лемм, а также неравенство $\| V_{1 t}\left(x_{2}\right)-$ $V_{2 t}\left(x_{2}\right) \|_{A} \leqslant d\left(V_{1 t}, V_{2 t}\right)$, которое является следствием определения метрики $d\left(V_{1 t}, V_{2 t}\right)$. Итак,

$$
d\left(\bar{V}_{1 t}, \bar{V}_{2 t}\right) \leqslant q_{2}(t) d\left(V_{1 t}, V_{2 t}\right),
$$

где $q_{2}(t)=\exp \left(\gamma_{3} t\right)\left[1+a_{5}(t)\right]+a_{1}(t)+2 a_{1}(t) a_{5}(t)$. Поэтому $q_{2}(0)=1, \dot{q}_{2}(0)=\gamma_{3}+2 K \leqslant \alpha_{N+1}+$ $3 K<0$ в силу предположений из условий теоремы 1 . Следовательно, $q_{2}(t)<1$ при $t>0$. Это обстоятельство позволяет сделать заключение о справедливости неравенства (11).

В результате получаем, что при $t \in\left[0, t_{7}\right], q_{2}(t)<1$ и оператор $G_{t}$-оператор сжатия. Положим теперь $t_{0}=\min \left(t_{1}, t_{2}, t_{3}, t_{4}, t_{5}, t_{6}, t_{7}\right)$. При таком выборе $t_{0}$ операторное уравнение $(10)$ при $t \in\left(0, t_{0}\right]$ имеет единственное решение $V_{t}(x) \in C_{Z}$, которое может быть найдено методом последовательных приближений, которые сходятся со скоростью геометрической прогрессии.

Для обоснования теоремы 1 в таком объеме осталось проверить справедливость двух утверждений:

1. операторное уравнение (10) имеет решение при всех $t>0$, а не только при $t \in\left(0, t_{0}\right]$; 
2. решение уравнения (10) не зависит от $t$, то есть

$$
V_{t_{1}}(x)=V_{t_{2}}(x)
$$

при любых $t_{1}, t_{2}$.

Для их проверки следует использовать полугрупповое свойство семейства операторов (1). В частности, из него вытекает полугрупповое свойство оператора $G_{t}$

$$
G_{t}(V(x))=G_{t_{1}} G_{t_{2}} V(x), \quad t=t_{1}+t_{2} .
$$

Откуда немедленно заключаем, что

$$
d\left(G_{t} V_{1}, G_{t} V_{2}\right) \leqslant q_{2}^{m}(t / m) d\left(V_{1}, V_{2}\right),
$$

где натуральное $m$ выбрано так, чтобы $t \in\left(0, t_{0}\right)$. Поэтому $G_{t}$ будет оператором сближения при всех $t>0$, а значит, операторное уравнение (10) имеет единственное решение при всех $t>0$.

Покажем, что неподвижная точка одна и также при всех $t>0$. Допустим, напротив, что это не так, и существуют такие $t_{1}, t_{2}>0$, что

$$
V_{t_{1}} \neq V_{t_{2}}
$$

Следовательно, $d\left(V_{t_{1}}, V_{t_{2}}\right)=\beta>0$. Возможно одно из двух:

1. числа $t_{1}$ и $t_{2}$ соизмеримы. Последнее означает, что существуют такие натуральные числа $k_{1}$, $k_{2}$, при которых имеем равенство $k_{1} t_{1}=k_{2} t_{2}$. В этом случае

$$
G_{k_{p} t_{p}}^{m} V_{t_{p}}=G_{t_{p}}^{k_{p} m} V_{t_{p}}=V_{t_{p}} \quad(p=1,2)
$$

при любом натуральном $m$.

Поэтому с одной стороны

$$
d\left(G_{k_{1} t_{1}}^{m} V_{1}, G_{k_{2} t_{2}}^{m} V_{2}\right)=d\left(V_{1}, V_{2}\right)=\beta>0,
$$

а с другой стороны

$$
d\left(G_{k_{1} t_{1}}^{m} V_{t_{1}}, G_{k_{2} t_{2}}^{m} V_{t_{2}}\right)=d\left(G_{k_{1} t_{1}}^{m} V_{t_{1}}, G_{k_{2} t_{2}}^{m} V_{t_{2}}\right) \rightarrow 0
$$

при $m \rightarrow \infty$, так как оператор $G_{t_{1}} G_{t_{2}}$ - оператор сближения.

2. Числа $t_{1}$ и $t_{1}$ несоизмеримы. Тогда, как хорошо известно, для любого достаточно малого $\delta>0$ можно указать такие натуральные $k_{1}, k_{2}>m(\delta)$, где $m(\delta) \rightarrow \infty$ при $\delta \rightarrow 0$, что $\left|k_{1} t_{1}-k_{2} t_{2}\right|<\delta$.

Положим $\Delta=k_{1} t_{1}-k_{2} t_{2}$ и будем для определенности считать, что $0<\Delta<\delta$. С одной стороны имеем

$$
d\left(G_{k_{1} t_{1}}^{m} V_{t_{1}}, G_{k_{2} t_{2}}^{m} V_{t_{2}}\right)=d\left(G_{t_{1}}^{m k_{1}} V_{t_{1}}, G_{t_{2}}^{m k_{2}} V_{t_{2}}\right)=d\left(V_{t_{1}}, V_{t_{2}}\right)=\beta>0,
$$

но $d\left(G_{k_{1} t_{1}}^{m} V_{t_{1}}, G_{k_{2} t_{2}}^{m} V_{t_{2}}\right)=d\left(G_{k_{2} t_{2}}\left[G_{\Delta} V_{t_{1}}\right], G_{k_{2} t_{2}} V_{t_{2}}\right)<\beta / 2$, если $k_{2}$ достаточно велико, то есть $m(\delta)$ велико, а $\delta$ - мало. Второе рассуждение использует то обстоятельство, что оператор $G_{t_{2}}-$ оператор сближения.

3. Доказательство теоремы об устойчивости инвариантного многообразия. Рассмотрим отображение (2) в переменных $x, y$. Последнее означает, что в этом разделе будем рассматривать семейство отображений (см. доказательство теоремы 1)

$$
x_{t}=T_{1}(t) x+X(t, x, y), \quad y_{t}=T_{2}(t) y+Y(t, x, y),
$$

где, как и ранее,

$$
\begin{aligned}
& X(t, x, y)=R_{1}(t, x, y+V(x)), \\
& Y(t, x, y)=R_{2}(t, x, y+V(x))+T_{2}(t) V(x)-V\left(T_{1}(t) x+X(t, x, y)\right) .
\end{aligned}
$$

Напомним, что выбор переменных $x, y$ означает, что

$$
Y(t, x, 0)=0, \quad\|Y(t, x, y)-Y(t, x, 0)\|_{A} \leqslant 2 a_{1}(t)\|y\|_{A} .
$$

Последние свойства гарантированы (см. второй раздел) при $t \in\left(0, t_{0}\right]$, где $t_{0}$ было выбрано в рамках доказательства первой части теоремы. Кроме того,

$$
\left\|y_{t}\right\|_{A} \leqslant\left\|T_{2}(t)\right\|\|y\|_{A}+\|Y(t, x, y)-Y(t, x, 0)\| \leqslant q_{3}(t)\|y\|_{A},
$$


где $q_{3}(t)=\exp \left(\gamma_{3} t\right)+2 a_{1}(t)$. При этом $q_{3}(0)=1, \dot{q}_{3}(0)=\gamma_{3}+2 K<0$, то есть $q_{3}(t)<1$ при $t \in\left(0, t_{*}\right)$, если $t_{*}$ - достаточно малая положительная постоянная. В частности, при любом $\tau \in\left(0, t_{*}\right)$ имеем

$$
q_{3}(\tau)=1+\left(\gamma_{3}+2 K\right) \tau+o(\tau) \leqslant 1+\frac{1}{2}\left(\gamma_{3}+2 K\right) \tau \leqslant \exp \left(\frac{1}{2}\left(\gamma_{3}+2 K\right) \tau\right) .
$$

Поэтому при $\tau \in\left(0, t_{*}\right]$ справедливо неравенство $\left\|y_{\tau}\right\|_{A} \leqslant \exp \left(\frac{1}{2}\left(\gamma_{3}+2 K\right) \tau\right)\|y\|_{A}$.

Пусть теперь $t>0$ и произвольно. Тогда $t=n t_{0}+\tau$, где $\tau \in\left(0, t_{0}\right)$ и $n \rightarrow \infty$, если $t \rightarrow \infty$ и наоборот, $t \rightarrow \infty$, если $n \rightarrow \infty$. Поэтому, во-первых, справедливо неравенство

$$
\left\|y_{t}\right\|_{A} \leqslant q_{3}(\tau) q_{3}^{n}\left(t_{0}\right)\|y\|_{A}
$$

a, во-вторых, $\lim _{t \rightarrow \infty}\left\|y_{t}\right\|_{A}=0$, так как $q_{3}\left(t_{0}\right)<1$.

Теорема 2 доказана, так как величина $\left\|y_{t}\right\|_{A}$ в переменных $x, y$ и есть расстояние от точки $(x, y)$ до инвариантного многообразия $M_{i n}$. При этом отметим, что убывание имеет экспоненциальную скорость, так как неравенство (13) можно переписать иначе

$$
\left\|y_{t}\right\|_{A}=\exp \left(\frac{1}{2}\left(\gamma_{3}+2 K\right) t\right)\|y\|_{A}, \quad \gamma_{3}+2 K<0
$$

4. Комментарии и замечания. В работе рассматривалась полугруппа нелинейных операторов в гильбертовом пространстве. Полученные результаты в некоторой степени близки к результатам основополагающей в данной тематике работы [18]. Следует отметить, что в этой работе рассматривалась полугруппа, порожденная нелинейным эволюционным уравнением, у которого линейная часть - симметричный линейный оператор (самосопряженный оператор в гильбертовом пространстве). Иными словами, линейная часть нелинейной полугруппы - полугруппа линейных ограниченных операторов класса $\left(C_{0}\right)$, производящий оператор которой симметричен. Последнее, как показано в данном разделе, необязательно и преодолимо. С другой стороны, полезно для приложений, так как требование симметричности для соответствующих линейных операторов выполнено далеко не всегда.

В данном разделе использован метод доказательства теоремы о существовании инвариантных многообразий, ведущий свое начало от работ Ф. Хартмана и С. Стернберга (см., например, [21]). Для изучения инерциальных многообразий он был использован в препринте [10].

Полученные результаты для динамических систем с бесконечномерным пространством близки к результатам, которые можно получить для $\gamma_{3}<0, \gamma_{2} \geqslant 0$. Инвариантное многообразие, соответствующее такому предположению, принято называть «центральным». В работах автора $[5,6]$ методы этого раздела были использованы для доказательства существования центральных многообразий для полугрупп (групп) нелинейных операторов, порожденных краевыми задачами для параболических (а также и гиперболических) дифференциальных уравнений с частными производными. Следует отметить, что аналогичная методика используется при изучении инвариантных многообразий иного типа, например, инвариантных торов $[2,3,9]$.

Рассмотренные выше полугруппы, как правило, порождены эволюционными уравнениями в вещественном гильбертовом пространстве, имеющими следующий вид

$$
\dot{u}=A u+F(u),
$$

где $u=u(t)$ - абстрактная функция со значениями в гильбертовом пространстве $H, A$-линейный замкнутый оператор, порождающий оператор полугруппы линейных операторов класса $\left(C_{0}\right)$ (или даже аналитической полугруппы). Нелинейный оператор $F(u)$ имеет в нуле порядок малости выше первого. В частности, $F(0)=0$. Иные свойства этого оператора подчас варьируются.

Приведем пример подходящих эволюционных уравнений $[14,17,19]$. В данной работе предполагается, что, во-первых, нелинейный оператор действует из пространства $H_{A}$ в $H$ и бесконечно дифференцируем по Фреше. Во-вторых, предполагается, что при каждом фиксированном $u \in H_{A}$ первая производная $F^{\prime}(u)$ является линейным ограниченным оператором из $H_{A}$ в $H$, допускает расширение до линейного оператора, который действует из $H$ в $H$. Близкие ограничения рассмотрены в работах $[17,19]$. Несколько иной класс эволюционных уравнений рассмотрен в работе [14], 
по задаче Коши для него также порождают подходящие для изучения полугруппы нелинейных операторов.

Тем не менее, следует отметить один важный аспект, возникающий при рассмотрении вопроса о существовании и свойствах инвариантных многообразий типа центральных или инерциальных. Уравнение (14) следует заменить на вспомогательное

$$
\dot{u}=A u+f(u),
$$

где вместо нелинейности $F(u)$ в правой части уравнения включена новая нелинейность $f(u)$. В свою очередь, нелинейный оператор $f(u)$ обладает следующими свойствами:

1. $f(u)=F(u), u \in S_{A}\left(r_{0}\right)$;

2. $f(u)=0, u \notin S_{A}\left(2 r_{0}\right)$;

3. $f(u)$ сохраняет достаточную гладкость (удовлетворяет условию Липшица) для всех $u \in H_{A}$.

Здесь $r_{0}$ - некоторая положительная постоянная.

Возможность такого переопределения носит обычно академический характер. Варианты таких переопределений указаны во многих работах (см., например, [12]). Если речь идет о динамических системах с бесконечномерным фазовым пространством, то, как хорошо известно, соответствующие построения возможны в гильбертовом пространстве или в банаховом пространстве с дифференцируемой нормой.

После соответствующих построений рассматривается задача Коши для уравнения (15), то есть это уравнение дополняется начальным условием

$$
u(0)=u_{0} \in H_{A},
$$

и порожденная задачей (15), (16) полугруппа нелинейных операторов, определенная уже с гарантией при всех $u \in H_{A}$ и $t \geqslant 0$.

Пусть $M_{\text {in }}$ инвариантное многообразие для решений задачи Коши $(15),(16)$, тогда это многообразие локально инвариантно для решений задачи Коши $(14),(16)$, которые принадлежат $S_{A}\left(r_{0}\right)$ и, следовательно, являются решениями вспомогательной задачи Коши (15), (16).

Добавим, что нелинейной полугруппе (1) или задачи Коши (15), (16) инвариантное многообразие $M_{\text {in }}$ существует и единственно, но для решений задачи (14), (16) оно, конечно, существует, но не обязательно единственно. При этом такая особенность для случая локально инвариантных многообразий может быть продемонстрирована уже и для обыкновенных дифференциальных уравнений. Соответствующий контрпример фактически стал известен после публикации А. М. Ляпуновым своей знаменитой работы [11]. Напомним этот пример. Рассмотрим систему дифференциальных уравнений

$$
\dot{x}=-\frac{1}{2} x^{3}, \quad \dot{y}=-y .
$$

У нее есть инвариантные многообразия вида

$$
y=c \exp \left(-1 / x^{2}\right), \text { если } x \neq 0, \quad y=0, \text { если } x=0, c \in \mathbb{R} .
$$

Впрочем, для обыкновенных дифференциальных уравнений существуют достаточные условия, реализация которых гарантирует единственность «центрального» многообразия.

Рассмотрим систему обыкновенных дифференциальных уравнений в $\mathbb{R}^{n+m}(n, m$ - натуральные числа)

$$
\begin{aligned}
& \dot{x}=A_{n} x+F_{n}(x, y), \\
& \dot{y}=B_{m} y+G_{m}(x, y) .
\end{aligned}
$$

Здесь $x=x(t) \in \mathbb{R}^{n}, y=y(t) \in \mathbb{R}^{m}$. Достаточно гладкие вектор-функции $F_{n}(x, y), G_{m}(x, y)$ имеют в нуле порядок малости выше первого. Наконец, матрица $A_{n}$ такая, что имеет С3, для которых справедливы неравенства $\operatorname{Re} \lambda_{j}(A) \geqslant 0$, а у матрицы $B_{m}$ все C3 лежат в полуплоскости, выделяемой неравенством $\operatorname{Re} \lambda_{k}(B)<0$.

Как уже неоднократно отмечалось в такой ситуации, у системы обыкновенных дифференциальных уравнений существует гладкое центральное (критическое) инвариантное многообразие $M_{i n}$, которое может быть задано уравнением $y=h(x), h(0)=0,\left.D_{x} h(x)\right|_{x=0}=0$, вектор-функция 
$h(x)$ по крайней мере в окрестности начала координат принадлежит классу $C^{m}-m$ раз непрерывно дифференцируемых вектор-функций.

Рассмотрим вспомогательную систему дифференциальных уравнений

$$
\dot{x}=-A_{n} x-F_{n}(x, h(x)),
$$

у которой, безусловно, есть нулевое СР $(x=0)$. Пусть оно асимптотически устойчиво. Тогда можно утверждать, что многообразие $M_{\text {in }}$ единственно $[7,8]$. Данный результат получен лишь для обыкновенных дифференциальных уравнений.

\section{СПИСОК ЛИТЕРАТУРЫ}

1. Гохберг И. И., Крейн М. Г. Введение в теорию несамосопряженных операторов. - М.: Наука, 1965.

2. Колесов А. Ю., Куликов А. Н., Розов Н. Х. Инвариантные торы одного класса точечных отображений: принцип кольца// Диффер. уравн. - 2003. - 39, № 5. - С. 584-601.

3. Колесов А. Ю., Куликов А. Н., Розов Н. Х. Инвариантные торы одного класса точечных отображений: сохранение инвариантного тора при возмущениях// Диффер. уравн. - 2003. - 39, № 6. - С. 738-753.

4. Крейн С. Г. Линейные дифференциальные операторы в банаховом пространстве. - М.: Наука, 1977.

5. Куликов $A$. Н. Интегральные многообразия гиперболических уравнений в случае, близком к критическому, одной пары чисто мнимых корней// Вестн. ЯрГУ. - 1975. - 13. - С. 94-117.

6. Куликов А. Н. О гладких инвариантных многообразиях полугруппы нелинейных операторов в банаховом пространстве// в кн.: Исследования по устойчивости и теории колебаний. - Ярославль, 1976. - C. $114-129$.

7. Куликов А. Н. Одно замечание о свойствах двумерных инвариантных многообразий// в кн.: Исследования по устойчивости и теории колебаний. - Ярославль, 1978. - С. 78-80.

8. Куликов А. Н. К вопросу о единственности инвариантного многообразия в критическом случае// в кн.: Исследования по устойчивости и теории колебаний. - Ярославль, 1979. - С. 81-85.

9. Куликов А. Н. Бифуркации инвариантных торов// в кн.: Исследования по устойчивости и теории колебаний. - Ярославль, 1983. - С. 112-117.

10. Куликов $A . H$. Инерциальные многообразия нелинейных автономных дифференциальных уравнений в гильбертовом пространстве/ Препринт № 85 Ин-та прикл. мат. им. М. В. Келдыша. - М., 1991.

11. Ляпунов А. М. Общая задача об устойчивости движения. - М.: Меркурий, 2000.

12. Марсден Дж., Мак-Кракен М. Бифуркации рождения цикла и ее приложения. - М.: Мир, 1980.

13. Наймарк М. А. Линейные дифференциальные операторы. - М.: Наука, 1965.

14. Соболевский П. Е. Об уравнениях параболического типа в банаховом пространстве// Тр. Моск. мат. о-ва. - 1961. - 10. - С. 297-350.

15. Хартман Ф. Обыкновенные дифференциальные уравнения. - М.: Мир, 1970.

16. Хилле Э., Филлипс Р. Обыкновенные дифференциальные уравнения. - М.: Мир, 1970.

17. Якубов С. Я. Разрешимость задачи Коши для абстрактных гиперболических уравнений второго порядка и их приложения// Тр. Моск. мат. о-ва. - 1970. - 23. - С. 37-60.

18. Foias G., Sell G. R., Temam R. Inertial manifolds for nonlinear evolutionary equations// J. Differ. Equ. - 1988. - 73. - P. 309-388.

19. Segal I. Nonlinear semigroups// Ann. Math. - 1963. - 78, № 2. — P. 339-364.

20. Slemrod M. Asymptotic behavior of $C_{0}$ semigroups as determined by the spectrum of the generator// Indiana Univ. Math. J. - 1977. - 25. - P. 783-792.

21. Sternberg S. Local contractions ant theorem of Poincaré// Am. J. Math. - 1957. - 79. - P. 809-824.

22. Zabcsyk J. A note on $C_{0}$ semigroups// Bull. Acad. Pol. Sci. Sér. Sci. Math. Astron. Phys. - 1975. — 27, № 8. - P. 895-898.

Куликов Анатолий Николаевич

Ярославский государственный университет им. П. Г. Демидова

E-mail: anat_kulikov@mail.ru 